MATHEMATICS OF COMPUTATION

Volume 66, Number 217, January 1997, Pages 31-44

S 0025-5718(97)00788-6

\title{
HOW ACCURATE IS THE STREAMLINE DIFFUSION FINITE ELEMENT METHOD?
}

\author{
GUOHUI ZHOU
}

\begin{abstract}
We investigate the optimal accuracy of the streamline diffusion finite element method applied to convection-dominated problems. For linear/bilinear elements the theoretical order of convergence given in the literature is either $O\left(h^{3 / 2}\right)$ for quasi-uniform meshes or $O\left(h^{2}\right)$ for some uniform meshes. The determination of the optimal order in general was an open problem. By studying a special type of meshes, it is shown that the streamline diffusion method may actually converge with any order within this range depending on the characterization of the meshes.
\end{abstract}

\section{INTRODUCTION}

We consider a model scalar convection-dominated convection-diffusion problem of the form

$$
\begin{array}{rlrl}
-\varepsilon \Delta u+u_{x}+u & =f & & \text { in } \Omega, \\
u=0 & & \text { on } \partial \Omega .
\end{array}
$$

Here, $\Omega$ is a bounded domain in $\mathbf{R}^{2}$, e.g., the unit square, with boundary $\partial \Omega$, while $0 \leq \varepsilon \ll 1$ is a small diffusion parameter.

The streamline diffusion finite element method (SDFEM) was proposed by Hughes et al. [2] and Johnson et al. [4] in order to cope with the usual instabilities caused by the convection term. It is capable of damping possible over- and undershootings of the discrete solution near the discontinuities while preserving higher order of convergence in regions where the solution is smooth. In fact, the SDFEM with linear or bilinear elements converges in $L^{2}$ with an order of $O\left(h^{3 / 2}\right)$ on general quasi-uniform meshes (see Johnson [3]), while the standard upwinding finite element method gives only $O(h)$.

A local pointwise error estimate of order $O\left(h^{5 / 4}\right)$ was given by Johnson et al. [5], which was later on improved by Niijima [7] to the order $O\left(h^{11 / 8}\right)$. Recently, it was shown in Zhou and Rannacher [13] that on streamline-oriented meshes the SDFEM even has the pointwise order of convergence $O\left(h^{2}|\log h|\right)$. The situation appeared somewhat confusing, as several test computations showed an unexpected $O\left(h^{2}\right)$-convergence, even on fairly general meshes.

Received by the editor June 1, 1995.

1991 Mathematics Subject Classification. Primary 65N30, 65B05, 76M10.

Key words and phrases. Convection-diffusion problems, streamline diffusion finite element method, structured meshes, superconvergence.

This work was supported by the Deutsche Forschungsgemeinschaft, SFB 359, Universität Heidelberg, Germany.

(C) 1997 American Mathematical Society 
In this paper, we try to clarify this question by studying the SDFEM on a special type of triangular meshes, which was introduced by Peterson [8] in the context of the discontinuous Galerkin method. He showed that on such meshes the order of convergence of the discontinuous Galerkin method is actually limited to $O\left(h^{3 / 2}\right)$. By our analysis it turns out that a similar effect occurs also for the SDFEM. We find an order of convergence in $L^{2}$ that may vary between $O\left(h^{3 / 2}\right)$ and $O\left(h^{2}\right)$ depending on a certain mesh parameter, while the pointwise error is bounded by $O\left(h^{3 / 2}\right)$ independent of the mesh parameter. These phenomena are confirmed by numerical tests. However, the extension of this result to quadrilateral meshes remains open.

\section{REVIEW OF SOME CONVERGENCE RESUlts}

First, we formulate the SDFEM and recall some well-known error estimates. Let $\Pi_{h}=\{e\}$ be quasi-uniform partitions of the polygonal domain $\Omega$ into triangles or (convex) quadrilaterals, where the largest diameter of all elements is denoted by $h$. Here, "quasi-uniform" means that the area of each element is bounded from below by $C h^{2}$ with some constant $C$ independent of $h$. On these meshes we define the finite element spaces

$$
\mathbf{V}_{h}=\left\{W \in \mathbf{H}_{0}^{1}(\Omega),\left.W\right|_{e} \text { is linear for a triangle or bilinear for a quadrilateral }\right\},
$$

where the term "bilinear" is to be understood in the usual isoparametric sense.

The SDFEM may be viewed as a modification of the standard Galerkin finite element method by using test functions of the form $V+\delta V_{x}$, with some small parameter $\delta$ of order $O(h)$. Accordingly, the discrete problem reads: Find $U \in \mathbf{V}_{h}$ such that

$$
\varepsilon(\nabla U, \nabla V)+\left(U_{x}+U, V+\delta V_{x}\right)=\left(f, V+\delta V_{x}\right), \quad \forall V \in \mathbf{V}_{h}
$$

The term $\left(\varepsilon \Delta U, \delta V_{x}\right)$ is neglected in our case. Since the parameter $\varepsilon$ is usually very small or even zero, the bilinear form defined by the left-hand side of (2.1) is only weakly positive definite, which causes problems in the local error analysis. To overcome this difficulty, Johnson et al. [5] proposed to replace $\varepsilon$ by an artificial diffusion coefficient $\varepsilon_{m}$ of size $O\left(h^{3 / 2}\right)$ to $O\left(h^{2}\right)$. Defining the bilinear form

$$
B(U, V)=\delta\left(U_{x}, V_{x}\right)+\varepsilon_{m}(\nabla U, \nabla V)+(1-\delta)\left(U_{x}, V\right)+(U, V),
$$

and the linear functional

$$
L(V)=\left(f, V+\delta V_{x}\right)
$$

we write the SDFEM in the compact form

$$
B(U, V)=L(V), \quad \forall V \in \mathbf{V}_{h}
$$

The energy form $B(\cdot, \cdot)$ is positive definite,

$$
B(V, V) \geq\|V\|^{2}, \quad \forall V \in \mathbf{H}_{0}^{1}(\Omega),
$$

with respect to the energy norm given by

$$
\|V\|^{2}=\left(\varepsilon_{m}+\delta\right)\left\|V_{x}\right\|^{2}+\varepsilon_{m}\left\|V_{y}\right\|^{2}+\|V\|^{2} .
$$

This implies that the discrete problem (2.4) possesses a unique solution $U \in \mathbf{V}_{h}$ and that the stability of the scheme is guaranteed. 
Further, we have the quasi-orthogonality relation

$$
B(u-U, V)=\operatorname{Per}(u, V), \quad \forall V \in \mathbf{V}_{h},
$$

with the truncation error

$$
\operatorname{Per}(u, V)=\left(\varepsilon \Delta u, \delta V_{x}\right)+\left(\varepsilon_{m}-\varepsilon\right)(\nabla u, \nabla V) .
$$

To obtain global $L^{2}$-error estimates, we use the standard argument for finite elements and introduce the linear/bilinear nodal interpolant $I_{h} u$ of the solution $u$. Clearly, there holds

$$
\left\|u-I_{h} u\right\| \leq C\left(\delta^{1 / 2} h+\varepsilon_{m}^{1 / 2} h+h^{2}\right)\left\|\nabla^{2} u\right\| .
$$

Setting $V=I_{h} u-U \in \mathbf{V}_{h}$ and using (2.5) and (2.7), we obtain

$$
\|V\|^{2} \leq B(V, V)=B\left(I_{h} u-u, V\right)+B(u-U, V)=B\left(I_{h} u-u, V\right)+\operatorname{Per}(u, V) .
$$

Further, using the estimates

$$
\begin{aligned}
B\left(I_{h} u-u, V\right) & \leq\left(\left\|I_{h} u-u\right\|+\delta^{-1 / 2}\left\|I_{h} u-u\right\|\right)\|V\| \\
& \leq C\left(\delta^{1 / 2} h+\varepsilon_{m}^{1 / 2} h+\delta^{-1 / 2} h^{2}\right)\left\|\nabla^{2} u\right\|\|V\|,
\end{aligned}
$$

and

$$
\operatorname{Per}(u, V) \leq C\left(\delta^{1 / 2} \varepsilon\left\|u_{x x}\right\|+\left|\varepsilon-\varepsilon_{m}\right|\|\Delta u\|\right)\|V\|,
$$

we conclude that

$$
\begin{aligned}
\|u-U\| \leq & C\left(\delta^{1 / 2} h+\varepsilon_{m}^{1 / 2} h+h^{2}+\delta^{-1 / 2} h^{2}\right)\left\|\nabla^{2} u\right\| \\
& +C\left(\delta^{1 / 2} \varepsilon\left\|u_{x x}\right\|+\left|\varepsilon-\varepsilon_{m}\right|\|\Delta u\|\right) .
\end{aligned}
$$

From this error estimate, we can infer that the optimal choice of $\delta$ is $\delta=O(h)$ and that the artificial diffusion $\varepsilon_{m}$ should satisfy $\varepsilon_{m} \leq C h^{3 / 2}$, to avoid a loss of accuracy. Then, we have the error estimate

$$
\|u-U\| \leq C h^{3 / 2}\left\|\nabla^{2} u\right\|
$$

where the constant $C$ does not depend on the diffusion coefficient $\varepsilon$. From this result for the energy norm, we immediately obtain also the $O\left(h^{3 / 2}\right)$-convergence for the $L^{2}$-norm $\|u-U\|$. The usual improvement of this to the optimal order of $O\left(h^{2}\right)$ by using a duality argument does not seem possible here, owing to the singular perturbation character of the problem. This leaves a gap of $O\left(h^{1 / 2}\right)$ in the order of convergence in the $L^{2}$-norm.

In view of the above discussion, it seems that on general meshes the SDFEM has the maximal order of convergence of $O\left(h^{3 / 2}\right)$. However, this may be improved on certain structured meshes. For instance, let a quadrilateral mesh be oriented in the streamline direction, i.e., parallel to the coordinate axes. For this, we recall the error expansion for the bilinear interpolation in Lin [6]. For an element $e$, we set $E(x)=\frac{1}{2}\left(\left(x-x_{e}\right)^{2}-h_{x}^{2}(e)\right), F(y)=\frac{1}{2}\left(\left(y-y_{e}\right)^{2}-h_{y}^{2}(e)\right)$, where $\left(x_{e}, y_{e}\right)$ is the center of the element and $2 h_{x}(e)$ and $2 h_{y}(e)$ are the edge lengths in the $x$ - and 
$y$-direction, respectively. Then, for $V=I_{h} u-U$ there holds

$$
\begin{aligned}
\delta\left(\left(I_{h} u-u\right)_{x}, V_{x}\right) & =\delta \sum_{e \in \Pi_{h}} \int_{e}\left\{F V_{x}-\frac{1}{3}\left(F^{2}\right)_{y} V_{x y}\right\} u_{x y y} d x d y \\
& \leq C \delta \sum_{e \in \Pi_{h}} h_{y}^{2} \int_{e}\left(\left|V_{x}\right|+h_{y}\left|V_{x y}\right|\right) u_{x y y} d x d y \\
& \leq C \delta^{1 / 2} h_{y}^{2}\left\|u_{x y y}\right\|\|V\|,
\end{aligned}
$$

and similarly,

$$
\varepsilon_{m}\left(\left(I_{h} u-u\right)_{y}, V_{y}\right) \leq C \varepsilon_{m}^{1 / 2} h_{x}^{2}\left\|u_{x x y}\right\|\|V\| .
$$

For estimating the convection term, we further require the mesh to be (almost) uniform in the streamline direction. Then, there holds

$$
\begin{aligned}
\left(\left(I_{h} u-u\right)_{x}, V\right)= & \sum_{e \in \Pi_{h}} \int_{e}\left(\frac{1}{6}\left(E^{2}\right)_{x} V_{x}-\frac{1}{3} h_{x}^{2} V\right) u_{x x x} d x d y \\
& +\sum_{e \in \Pi_{h}} \int_{e}\left(F\left(V-E_{x} V_{x}\right)-\frac{1}{3}\left(F^{2}\right)_{y}\left(V_{y}-E_{x} V_{x y}\right)\right) u_{x y y} d x d y \\
\leq & C h_{x}^{2}\left\|u_{x x x}\right\|\|V\|+C h_{y}^{2}\left\|u_{x y y}\right\|\|V\| \\
\leq & C\left(h_{x}^{2}+h_{y}^{2}\right)\left(\left\|u_{x x x}\right\|+\left\|u_{x y y}\right\|\right)\|V\| .
\end{aligned}
$$

Further,

$$
\left(I_{h} u-u, V\right) \leq C\left(h_{x}^{2}\left\|u_{x x}\right\|+h_{y}^{2}\left\|u_{y y}\right\|\right)\|V\| .
$$

Using these estimates, instead of (2.8), we now have

$$
B\left(I_{h} u-u, V\right) \leq C h^{2}\left(\delta^{1 / 2}+\varepsilon_{m}^{1 / 2}+1\right)\left(\left\|\nabla^{2} u\right\|+\left\|\nabla^{3} u\right\|\right)\|V\| .
$$

This gives us the estimate

$$
\begin{aligned}
\left\|I_{h} u-U\right\| \leq & C h^{2}\left(\delta^{1 / 2}+\varepsilon_{m}^{1 / 2}+1\right)\left(\left\|\nabla^{2} u\right\|+\left\|\nabla^{3} u\right\|\right) \\
& +C\left(\delta \varepsilon\left\|u_{x x x}\right\|+\left|\varepsilon-\varepsilon_{m}\right|\|\Delta u\|\right),
\end{aligned}
$$

from which we infer, for any choice of $\delta$ and for $\varepsilon \leq \varepsilon_{m}=C h^{2}$, that

$$
\|u-U\| \leq\left\|u-I_{h} u\right\|+\left\|I_{h} u-U\right\| \leq C h^{2}\|u\|_{H^{3}(\Omega)} .
$$

We note that for this global error estimate, no lower bounds for $\delta$ and $\varepsilon_{m}$ are needed. But for estimating the local $L^{2}$-error or the pointwise error, the sizes of $\delta$ and $\varepsilon_{m}$ have to satisfy $\delta=O\left(h_{x}\right)$ and $\varepsilon_{m}=O\left(h_{y}^{2}\right)$, see [13]. Such a superconvergence result can also be established for three-directional triangular meshes by using the techniques proposed in Blum et al. [1]. For the discontinuous Galerkin method, similar superconvergence results were also achieved in Richter [9] under some mesh conditions.

The first local pointwise error estimate for the SDFEM was given in [5],

$$
\left|(u-U)\left(x_{0}, y_{0}\right)\right| \leq C h^{5 / 4}|\log h|^{3 / 2}\|u\|_{H^{2}\left(\Omega_{0}\right)}+C h^{\nu},
$$

for quasi-uniform meshes and for any $\nu \geq 2$, where

$$
\Omega_{0}=\left\{(x, y): x-x_{0} \leq C h|\log h|,\left|y-y_{0}\right| \leq C h^{3 / 4}|\log h|\right\} .
$$


The proof is based on local bounds for the discrete Green functions. This pointwise convergence result was later improved in [7] to

$$
\left|(u-U)\left(x_{0}, y_{0}\right)\right| \leq C h^{11 / 8}|\log h|\|u\|_{H^{2}\left(\Omega_{0}\right)}+C h^{\nu} .
$$

Already in [5], it was conjectured that the SDFEM should converge pointwise with the order $O\left(h^{3 / 2}\right)$ or even $O\left(h^{2}\right)$. This could be confirmed in [13], at least on certain structured meshes. On streamline-oriented and uniform meshes, the estimate (2.13) was improved to

$$
\left|(u-U)\left(x_{0}, y_{0}\right)\right| \leq C h^{2}|\log h|\|u\|_{H^{3}\left(\Omega_{0}\right)}+C h^{\nu},
$$

with

$$
\Omega_{0}=\left\{(x, y): x-x_{0} \leq C h|\log h|,\left|y-y_{0}\right| \leq C h|\log h|\right\} .
$$

This result not only improved on the order of convergence, but also reduced the width of the dependence subdomain $\Omega_{0}$. The reduction of the crosswind smear to $O(h|\log h|)$ was also given in [10] by a stencil analysis.

In [13], the SDFEM has been tested for various model problems on different types of meshes. Surprisingly, in almost all cases an $O\left(h^{2}\right)$-convergence was observed, even though the uniformity condition on the mesh was violated. This led to the impression that the SDFEM would indeed converge with the optimal order of $O\left(h^{2}\right)$ on general quasi-uniform meshes. However, this was finally disproved by a test calculation on a very special triangular mesh introduced in [8] for an investigation of the convergence property of the discontinuous Galerkin method for convection problems. This special mesh violates the mesh condition proposed by Richter [9]. This pathological mesh will be described in the next section and a detailed theoretical analysis will be given, which explains the reason for the order reduction in the SDFEM.

\section{THE SDFEM ON A SPECIAL MESH}

We now investigate the convergence of the SDFEM on the special meshes proposed in [8]. We start with a simple convection problem, a similar form of which was also considered in [8],

$$
\begin{array}{ll}
u_{y}+u=x^{2} & \text { in } \Omega, \\
u(x, 0)=x^{2} & \text { for } 0 \leq x \leq 1 .
\end{array}
$$

The exact solution is $u(x, y)=x^{2}$. The SDFEM is applied to this problem on meshes as shown in Figure 1, where additional vertical lines are inserted, with varying number $m \approx h^{-\sigma}, 0 \leq \sigma \leq 1$. For the mesh size $h=2^{-N}$, Table 1 shows an unexpected reduction of the convergence order in $L^{2}$, depending on the exponent $\sigma$.

Remark 1. The computational results shown in Table 1 suggest that the order of convergence should depend on the number $\sigma$ of inserted lines like

$$
\|u-U\| \leq \begin{cases}C h^{2-2 \sigma / 3} & \text { for } 0 \leq \sigma \leq \frac{3}{4} \\ C h^{2 \sigma} & \text { for } \frac{3}{4} \leq \sigma \leq 1\end{cases}
$$

In Table 2, the corresponding order of convergence in $L^{\infty}$ seems independent of $m$. The order of pointwise convergence is reduced to $O\left(h^{3 / 2}\right)$ even if only one vertical line is inserted. However, in both cases, the superconvergence is again 

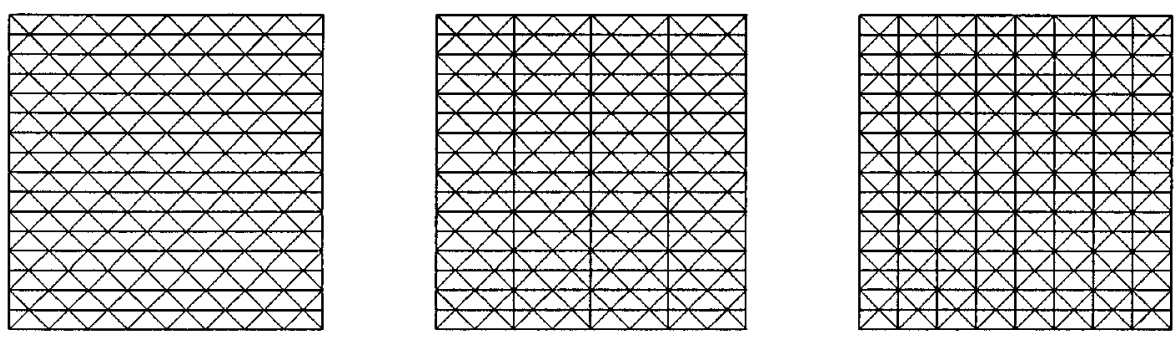

FiguRE 1. The original mesh and two modified meshes with $m=3$ and $m=h^{-1}$

TABLE 1. The $L^{2}$-error and convergence order for problem (3.1)

\begin{tabular}{|l|r|r|r|r|r|r|r|}
\hline$N$ & 3 & 4 & 5 & 6 & 7 & 8 & 9 \\
\hline$m=h^{-1}$ & $4.12 \mathrm{e}-3$ & $1.07 \mathrm{e}-3$ & $2.73 \mathrm{e}-4$ & $6.88 \mathrm{e}-5$ & $1.73 \mathrm{e}-5$ & $4.33 \mathrm{e}-6$ & $1.08 \mathrm{e}-6$ \\
\cline { 2 - 8 } & order & 1.95 & 1.97 & 1.99 & 1.99 & 2.00 & 2.00 \\
\hline$m \approx 0.25 h^{-1}$ & $4.91 \mathrm{e}-3$ & $1.48 \mathrm{e}-3$ & $4.59 \mathrm{e}-4$ & $1.55 \mathrm{e}-4$ & $5.10 \mathrm{e}-5$ & $1.52 \mathrm{e}-5$ & $4.20 \mathrm{e}-6$ \\
\cline { 2 - 8 } & order & 1.73 & 1.69 & 1.57 & 1.60 & 1.75 & 1.86 \\
\hline$m \approx h^{-3 / 4}$ & $4.40 \mathrm{e}-3$ & $1.36 \mathrm{e}-3$ & $3.96 \mathrm{e}-4$ & $1.32 \mathrm{e}-4$ & $4.34 \mathrm{e}-5$ & $1.52 \mathrm{e}-5$ & $5.39 \mathrm{e}-5$ \\
\cline { 2 - 8 } & order & 1.69 & 1.78 & 1.58 & 1.60 & 1.51 & 1.50 \\
\hline$m \approx h^{-1 / 2}$ & $4.98 \mathrm{e}-3$ & $1.48 \mathrm{e}-3$ & $4.28 \mathrm{e}-4$ & $1.18 \mathrm{e}-4$ & $3.55 \mathrm{e}-5$ & $1.13 \mathrm{e}-5$ & $3.63 \mathrm{e}-6$ \\
\cline { 2 - 8 } & order & 1.75 & 1.79 & 1.86 & 1.73 & 1.65 & 1.64 \\
\hline$m \approx h^{-2 / 5}$ & $4.91 \mathrm{e}-2$ & $1.38 \mathrm{e}-3$ & $3.67 \mathrm{e}-4$ & $1.04 \mathrm{e}-4$ & $3.06 \mathrm{e}-5$ & $9.04 \mathrm{e}-6$ & $2.75 \mathrm{e}-6$ \\
\cline { 2 - 8 } & order & 1.83 & 1.91 & 1.82 & 1.76 & 1.76 & 1.72 \\
\hline$m=1$ & $4.62 \mathrm{e}-3$ & $1.21 \mathrm{e}-3$ & $3.11 \mathrm{e}-4$ & $8.00 \mathrm{e}-5$ & $2.06 \mathrm{e}-5$ & $5.32 \mathrm{e}-6$ & $1.38 \mathrm{e}-5$ \\
\cline { 2 - 8 } & order & 1.93 & 1.96 & 1.96 & 1.96 & 1.95 & 1.95 \\
\hline
\end{tabular}

TABLE 2. The maximum error and convergence order for problem (3.1)

\begin{tabular}{|l|r|r|r|r|r|r|r|}
\hline$N$ & 3 & 4 & 5 & 6 & 7 & 8 & 9 \\
\hline$m=h^{-1}$ & $6.41 \mathrm{e}-3$ & $1.64 \mathrm{e}-3$ & $4.16 \mathrm{e}-4$ & $1.05 \mathrm{e}-4$ & $2.63 \mathrm{e}-5$ & $6.58 \mathrm{e}-6$ & $1.65 \mathrm{e}-6$ \\
\cline { 2 - 8 } & order & 1.97 & 1.98 & 1.99 & 2.00 & 2.00 & 2.00 \\
\hline$m \approx 0.25 h^{-1}$ & $9.85 \mathrm{e}-3$ & $3.06 \mathrm{e}-3$ & $9.32 \mathrm{e}-4$ & $3.60 \mathrm{e}-4$ & $1.21 \mathrm{e}-4$ & $3.69 \mathrm{e}-5$ & $1.04 \mathrm{e}-5$ \\
\cline { 2 - 8 } & order & 1.69 & 1.72 & 1.37 & 1.57 & 1.71 & 1.83 \\
\hline$m \approx h^{-3 / 4}$ & $1.00 \mathrm{e}-2$ & $2.64 \mathrm{e}-3$ & $9.01 \mathrm{e}-4$ & $3.08 \mathrm{e}-4$ & $1.01 \mathrm{e}-4$ & $3.69 \mathrm{e}-5$ & $1.35 \mathrm{e}-5$ \\
\cline { 2 - 8 } & order & 1.92 & 1.55 & 1.55 & 1.61 & 1.45 & 1.45 \\
\hline$m \approx h^{-1 / 2}$ & $9.63 \mathrm{e}-4$ & $3.06 \mathrm{e}-3$ & $9.06 \mathrm{e}-4$ & $3.42 \mathrm{e}-4$ & $1.32 \mathrm{e}-4$ & $5.05 \mathrm{e}-5$ & $1.87 \mathrm{e}-5$ \\
\cline { 2 - 8 } & order & 1.65 & 1.76 & 1.41 & 1.37 & 1.39 & 1.43 \\
\hline$m \approx h^{-2 / 5}$ & $9.85 \mathrm{e}-4$ & $2.78 \mathrm{e}-3$ & $8.17 \mathrm{e}-4$ & $3.38 \mathrm{e}-4$ & $1.32 \mathrm{e}-4$ & $5.04 \mathrm{e}-5$ & $1.87 \mathrm{e}-5$ \\
\cline { 2 - 8 } & order & 1.83 & 1.77 & 1.27 & 1.36 & 1.39 & 1.43 \\
\hline$m=1$ & $8.73 \mathrm{e}-4$ & $2.47 \mathrm{e}-3$ & $8.17 \mathrm{e}-4$ & $3.38 \mathrm{e}-4$ & $1.33 \mathrm{e}-4$ & $5.04 \mathrm{e}-5$ & $1.87 \mathrm{e}-5$ \\
\cline { 2 - 8 } & order & 1.82 & 1.60 & 1.27 & 1.35 & 1.40 & 1.43 \\
\hline
\end{tabular}

recovered for the extreme case $m=h^{-1}$. Note that this mesh is still not a complete crisscross mesh. Figure 2 shows the error behavior for problem (3.1). 

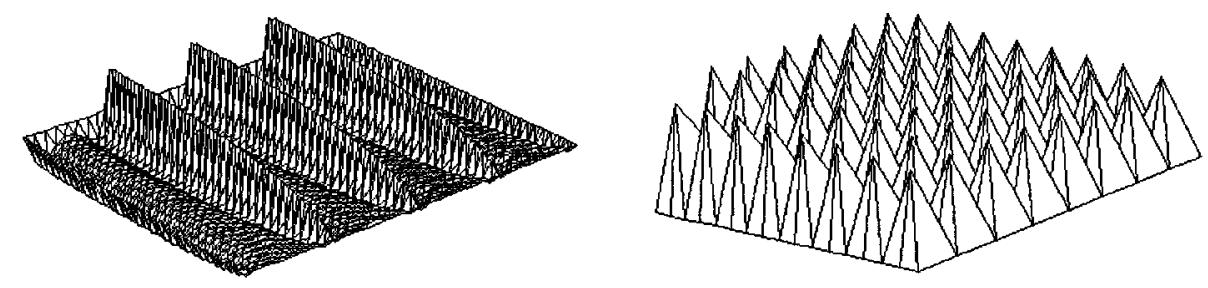

FiguRE 2. The error behavior of the SDFEM with $m=3$ and $m=h^{-1}$ for problem (3.1)

The above effect crucially depends on the orientation of the mesh. To demonstrate this, we consider another "rotated" problem on the same meshes,

$$
\begin{array}{ll}
u_{x}+u=y^{2} & \text { in } \Omega, \\
u(0, y)=y^{2} & \text { for } 0 \leq y \leq 1,
\end{array}
$$

with the exact solution $u(x, y)=y^{2}$. The computational results show that in this case the error is not affected by the inserted lines and the convergence order is $O\left(h^{2}\right)$ in both norms. In the next section, we will try to explain the reasons for these phenomena.

\section{ERROR ANALYSIS FOR THE SPECIAL MESHES}

From the previous section, we see for $V=I_{h} u-U$ that

$$
\|V\|^{2} \leq B(V, V)=B\left(I_{h} u-u, V\right)+B(u-U, V)=B\left(I_{h} u-u, V\right)+\operatorname{Per}(u, V) .
$$

Therefore, the error estimate for the SDFEM essentially reduces to the estimate for the interpolation error term $B\left(I_{h} u-u, V\right)$. To estimate the convection term, we need an error expansion lemma from Zhou and Lin [11].

Lemma 1. Let $I_{h} u$ be the linear nodal interpolant of the function $u$ on a triangular element $e$ and let $V$ be any function in $H^{1}(e)$. There holds the expansion estimate

$$
\int_{e}\left(u-I_{h} u\right) V_{y} d x d y=-\frac{h^{2}}{24} \oint_{\partial e}\left(\sum_{i=1}^{3} \lambda_{i}^{2} D_{i}^{2} u\right) V n_{y} d s+O\left(h^{2}\|u\|_{H^{3}(e)}\|V\|_{e}\right),
$$

where $D_{i}$ denotes the directional derivative along the side $s_{i}$ of $e$ whose length is $h_{i}=\lambda_{i} h$.

Summing the expansion (4.1) over all elements, we obtain

$$
\left(\left(u-I_{h} u\right), V_{y}\right)=-\frac{h^{2}}{24} \sum_{e \in \Pi_{h}} \oint_{\partial e}\left(\sum_{i=1}^{3} \lambda_{i}^{2} D_{i}^{2} u\right) V n_{y} d s+O\left(h^{2}\|u\|_{H^{3}}\|V\|\right) .
$$

Consider now one level of elements in the mesh shown in Figure 3, $y_{n}-\frac{1}{2} h \leq$ $y \leq y_{n}+\frac{1}{2} h$. Suppose that a vertical line is inserted at $x=x_{m}$. There are only 6 different types of elements to be analyzed, which are numbered by 1 to 6 . By $\vec{k}$ and $\vec{l}$, we denote the directions $(-1,1)$ and $(1,1)$, respectively. For element $e$, we denote by $\partial e_{l}, \partial e_{k}, \partial e_{x}$ and $\partial e_{y}$ the sides parallel to the indexed directions. 


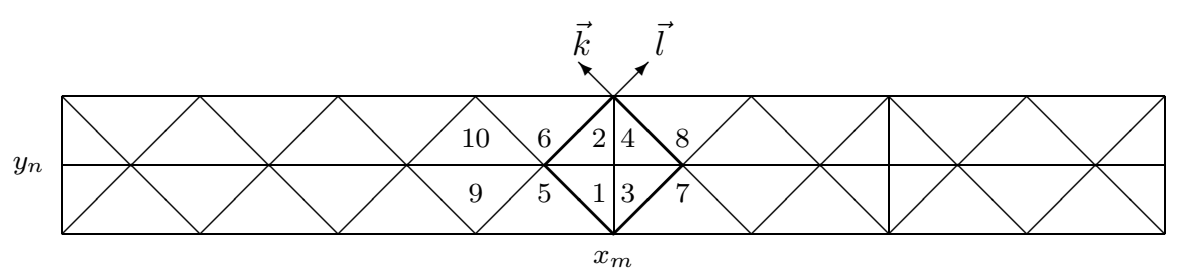

Figure 3. One level of elements

First, let us analyze the error in elements $e_{1}, \ldots, e_{6}$. It is easy to see that on elements $e_{5}$ and $e_{6}$ there holds

$$
\sum_{i=1}^{3} \lambda_{i}^{2} D_{i}^{2} u=u_{x x}+\frac{1}{2} u_{l l}+\frac{1}{2} u_{k k}=u_{x x}+\frac{1}{2} \Delta u,
$$

where we used the fact that the Laplace operator is invariant under rotations. On elements $e_{1}$ and $e_{4}$, we have

$$
\sum_{i=1}^{3} \lambda_{i}^{2} D_{i}^{2} u=\frac{1}{4} u_{x x}+\frac{1}{4} u_{y y}+\frac{1}{2} u_{k k}=\frac{1}{4} \Delta u+\frac{1}{2} u_{k k},
$$

and on elements $e_{2}$ and $e_{3}$,

$$
\sum_{i=1}^{3} \lambda_{i}^{2} D_{i}^{2} u=\frac{1}{4} u_{x x}+\frac{1}{4} u_{y y}+\frac{1}{2} u_{l l}=\frac{1}{4} \Delta u+\frac{1}{2} u_{l l} .
$$

Since the integrands on elements $e_{9}$ and $e_{10}$ are the same, the line integral on the edge between elements $e_{9}$ and $e_{10}$ is zero. By the same argument, the line integrals on the edges between $e_{9}$ and $e_{5}$ and between $e_{10}$ and $e_{6}$ vanish, too. In other words, away from the inserted vertical lines, all line integrals disappear. Now, we investigate the effect of the inserted vertical lines. For simplicity of notation, we set $T\left(x_{m}, y_{n}\right)=\bigcup_{i=1}^{4} e_{i}, \partial T_{1}=e_{1} \cap e_{5}, \partial T_{2}=e_{2} \cap e_{6}, \partial T_{3}=e_{3} \cap e_{7}, \partial T_{4}=e_{4} \cap e_{8}$, $\partial T_{12}=e_{1} \cap e_{2}, \partial T_{34}=e_{3} \cap e_{4}, \partial T_{13}=e_{1} \cap e_{3}, \partial T_{24}=e_{2} \cap e_{4}$.

First, let us look at the line integral on the edge $\partial T_{1}$. By subtracting (4.4) from (4.3), this integral becomes

$$
\frac{1}{\sqrt{2}} \int_{\partial T_{1}}\left(u_{x x}+\frac{1}{4} \Delta u-\frac{1}{2} u_{k k}\right) V d s=\frac{1}{\sqrt{2}} \int_{\partial T_{1}}\left(u_{x x}+\frac{1}{4} u_{x y}\right) V d s .
$$

It is not difficult to see that the line integral on the edge $\partial T_{4}$ has the same integrand as the above, but with negative sign. Subtracting (4.3) from (4.5), we have the line integral on the edge $\partial T_{2}$,

$$
\frac{1}{\sqrt{2}} \int_{\partial T_{2}}\left(-u_{x x}-\frac{1}{4} \Delta u+\frac{1}{2} u_{l l}\right) V d s=\frac{1}{\sqrt{2}} \int_{\partial T_{2}}\left(-u_{x x}+\frac{1}{4} u_{x y}\right) V d s .
$$

The line integral on the edge $\partial T_{3}$ has the same form, but with opposite sign. On the line $\partial T_{12}$, we have

$$
\int_{\partial T_{12}}\left(u_{k k}-u_{l l}\right) V d s=-\int_{\partial T_{12}} u_{x y} V d s
$$


while on $\partial T_{34}$ the integral just changes sign. Finally, we have $n_{y}=0$ along the vertical line. Summing over all the elements, we thus obtain

$$
\begin{aligned}
\left(\left(u-I_{h} u\right), V_{y}\right)= & -\frac{h^{2}}{24 \sqrt{2}} \sum_{T}\left(\int_{\partial T_{1}}-\int_{\partial T_{2}}+\int_{\partial T_{3}}-\int_{\partial T_{4}}\right) u_{x x} V d s \\
& -\frac{h^{2}}{96 \sqrt{2}} \sum_{T}\left(\int_{\partial T_{1}}+\int_{\partial T_{2}}-\int_{\partial T_{3}}-\int_{\partial T_{4}}\right) u_{x y} V d s \\
& +\frac{h^{2}}{24} \sum_{T}\left(\int_{\partial T_{12}}-\int_{\partial T_{34}}\right) u_{x y} V d s \\
& +O\left(h^{2}\|u\|_{H^{3}}\|V\|\right),
\end{aligned}
$$

where here and below the summation for $T$ runs over all crisscross elements $T$ defined above.

Now, we consider a special case with $u_{x y} \equiv 0$. The second and the third term on the right-hand side of the above expansion vanish. Next, we consider the crisscross strip at $x=x_{m}$. The pieces of line integrals of type $\partial T_{1}$ and $\partial T_{2}$ go zigzag from the bottom to the top, with changing signs. Setting $P_{2 j}=\left(x_{m}, y_{2 j}\right)$ for $j=0, \ldots, N$ and $P_{2 j+1}=\left(x_{m}-\frac{1}{2} h, y_{2 j+1}\right)$, for $j=0, \ldots, N-1$, we use the trapezoidal rule on the equidistant intervals $\left(P_{i}, P_{i+1}\right)$ to get

$$
\begin{aligned}
\left|\sum_{i=0}^{2 N-1} \int_{P_{i}}^{P_{i+1}}(-1)^{i} u_{x x} V d s\right| \leq & \frac{h}{2} \sum_{i=0}^{2 N-1}(-1)^{i}\left(u_{x x}\left(P_{i}\right) V\left(P_{i}\right)+u_{x x}\left(P_{i+1}\right) V\left(P_{i+1}\right)\right) \mid \\
& +C h^{2} \sum_{i=0}^{2 N-1} \int_{P_{i}}^{P_{i+1}}\left|\left(u_{x x} V\right)_{s s}\right| d s \\
\leq & C h^{2} \int_{P_{0}}^{P_{2 N}}\left(\left|u_{x x s}\right|\left|V_{s}\right|+\left|u_{x x s s}\right||V|\right) d s,
\end{aligned}
$$

where $P_{0}$ and $P_{2 N}$ are located on the boundary $\partial \Omega$ at which $V$ vanishes. The same can be done for the line integrals of type $\partial T_{3}$ and $\partial T_{4}$. Using the inverse property, we simplify the estimate:

$$
\begin{aligned}
\left|\left(\left(u-I_{h} u\right), V_{y}\right)\right| & \leq C h^{3} \sum \int_{P_{0}}^{P_{2 N}}\left(\left|u_{x x s}\right|+h\left|u_{x x s s}\right|\right)|V| d s+C h^{2}\|u\|_{H^{3}}\|V\| \\
& \leq C\left(h^{2}\|u\|_{H^{3}}+h^{3}\|u\|_{H^{4}}\right)\|V\| .
\end{aligned}
$$

Similarly, we can expand the error

$$
\begin{aligned}
\left(\left(u-I_{h} u\right), V_{x}\right)= & \frac{h^{2}}{24 \sqrt{2}} \sum_{T}\left(\int_{\partial T_{1}}+\int_{\partial T_{2}}-\int_{\partial T_{3}}-\int_{\partial T_{4}}\right) u_{x x} V d s \\
& -\frac{h^{2}}{96 \sqrt{2}} \sum_{T}\left(\int_{\partial T_{1}}-\int_{\partial T_{2}}+\int_{\partial T_{3}}-\int_{\partial T_{4}}\right) u_{x y} V d s \\
& +\frac{h^{2}}{24} \sum_{T}\left(\int_{\partial T_{13}}-\int_{\partial T_{24}}\right) u_{x y} V d s \\
& +O\left(h^{2}\|u\|_{H^{3}}\|V\|\right) .
\end{aligned}
$$

If we assume in this case that $u_{x x} \equiv 0$, we can prove in the same way that

$$
\left|\left(\left(u-I_{h} u\right), V_{x}\right)\right| \leq C\left(h^{2}\|u\|_{H^{3}}+h^{3}\|u\|_{H^{4}}\right)\|V\| .
$$


Remark 2. The error estimates (4.7) and (4.9) show that the standard finite element method for convection problems converges in the $L^{2}$-norm with the order of $O\left(h^{2}\right)$ on Peterson's meshes for any number of inserted lines, even though it lacks stability in the energy norm. A novice reader may be reminded that the standard FEM is useless if there are boundary layers. Test computations also confirm this superconvergence property. On the other hand, the test computations also show that the condition $u_{x y} \equiv 0$ or $u_{x x} \equiv 0$ is not necessary for the above results.

To estimate the diffusion terms $\left(\left(u-I_{h} u\right)_{x}, V_{x}\right)$ and $\left(\left(u-I_{h} u\right)_{y}, V_{y}\right)$, we need an error expansion result from [1], which is summarized in the following lemma.

Lemma 2. Let $I_{h} u$ be the linear nodal interpolant of the function $u$ on a triangular element $e$ and $V$ be any function in $\mathbf{V}_{h}(e)$. There holds the error expansion

$$
\begin{aligned}
\int_{e}\left(I_{h} u\right. & -u)_{\mu} V_{\nu} d x d y \\
= & \frac{h^{4}}{24 A} \sum_{i=1}^{3} \int_{s_{i}} D_{i} V\left(\lambda_{i+1}^{4} n_{\mu}^{i+1} n_{\nu}^{i+1} D_{i+1}^{2} u-\lambda_{i}^{3} \lambda_{i+2} n_{\mu}^{i} n_{\nu}^{i+2} D_{i}^{2} u\right) d s \\
& +\frac{h^{4}}{24 A} \sum_{i=1}^{3} \int_{s_{i}} V\left(\lambda_{i+2}^{4} n_{\mu}^{i+2} n_{\nu}^{i+2} D_{i} D_{i+2}^{2} u-\lambda_{i}^{3} \lambda_{i+1} n_{\mu}^{i} n_{\nu}^{i} D_{i+1} D_{i}^{2} u\right) d s \\
& +\frac{h^{6} \lambda_{1} \lambda_{2} \lambda_{3}}{48 A^{2}} \sum_{i=1}^{3} \int_{e} \lambda_{i}^{3} n_{\mu}^{i} n_{\nu}^{i} D_{1} D_{2} D_{3} D_{i} u V d x d y \\
& +O\left(h^{2}\|u\|_{H^{4}(e)}\|V\|_{e}\right),
\end{aligned}
$$

where $A$ denotes the area of the element $e$ and $D_{i}$ denotes the directional derivative along the side $s_{i}$ whose length is $h_{i}=\lambda_{i} h$ and on which the $\mu$-component $(x$ or $y)$ of the outer normal is $n_{\mu}^{i}$. The index $i+1$ is used $\bmod (3)$.

Using this expansion, we obtain through careful calculations that

$$
\begin{aligned}
\left(\left(u-I_{h} u\right)_{x}, V_{x}\right)= & -\frac{h^{2}}{24} \sum_{T}\left(\int_{\partial T_{12}}-\int_{\partial T_{34}}\right) V_{x} u_{x x} d x \\
& +C\|V\|\left(h\|u\|_{H^{3}}+C h^{2}\|u\|_{H^{4}}\right) .
\end{aligned}
$$

Combining this with the expansion (4.9), we have proved the following theorem.

Theorem 1. For the problem

$$
\begin{aligned}
-\varepsilon \Delta u+u_{x}+u & =f & & \text { in } \Omega, \\
u & =g & & \text { on } \partial \Omega,
\end{aligned}
$$

with $u_{x x} \equiv 0$, the Peterson meshes do not affect the superconvergence of the SDFEM:

$$
\|u-U\| \leq C h^{2}\left(\|u\|_{H^{3}}+h\|u\|_{H^{4}}\right) .
$$

Remark 3. As before, the test computations show that the condition $u_{x x} \equiv 0$ is not necessary for the superconvergence, it is only used for technical reasons. 
Now, we consider another model problem,

$$
\begin{aligned}
-\varepsilon \Delta u+u_{y}+u=f & & \text { in } \Omega, \\
u=g & & \text { on } \partial \Omega,
\end{aligned}
$$

with $\varepsilon \geq 0$. In the SDFEM we take $\delta=C h$ and $\varepsilon_{m}=C h^{\alpha}$ with $\frac{3}{2} \leq \alpha \leq 2$. The associated energy norm is

$$
\|V\|^{2}=h\left\|V_{y}\right\|^{2}+h^{\alpha}\left\|V_{x}\right\|^{2}+\|V\|^{2} .
$$

Exchanging $x$ with $y$ in (4.10), we can get by a lengthy analysis

$$
\begin{aligned}
\left(\left(u-I_{h} u\right)_{y}, V_{y}\right)= & -\frac{h^{2}}{8} \sum_{T}\left(\int_{\partial T_{13}}-\int_{\partial T_{24}}\right) V_{y} u_{x x} d y \\
& +\frac{h^{2}}{24} \sum_{T}\left(\int_{\partial T_{12}}-\int_{\partial T_{34}}\right) V_{x}\left(3 u_{x x}-u_{y y}\right) d x \\
& +C\|V\|\left(h\|u\|_{H^{3}}+h^{2}\|u\|_{H^{4}}\right) .
\end{aligned}
$$

Since $V_{y}$ is theoretically not continuous at the nodal point, we cannot use the trapezoidal rule as before. We estimate as follows:

$$
\begin{aligned}
\delta\left|\left(\left(u-I_{h} u\right)_{y}, V_{y}\right)\right| \leq & C h^{2} \sum_{T}\left(\int_{T}\left|V_{y}\right|\left|u_{x x}\right| d x d y+\int_{T}\left|V_{x}\right|\left|3 u_{x x}-u_{y y}\right| d x d y\right) \\
& +C\|V\|\left(h^{2}\|u\|_{H^{3}}+h^{3}\|u\|_{H^{4}}\right) .
\end{aligned}
$$

By $\Omega_{1}$ we denote all the strips containing the inserted vertical lines. Noting that each strip is only of width $h$, we have for a number $m \approx h^{-\sigma}$ of vertical lines

$$
\operatorname{meas}\left(\Omega_{1}\right)=h m=C h^{1-\sigma} .
$$

Using this, we obtain

$$
\begin{aligned}
\delta \mid((u- & \left.\left.I_{h} u\right)_{y}, V_{y}\right) \mid \\
\leq & C h^{(5-\sigma) / 2}\left(\left\|V_{y}\right\|\left\|u_{x x}\right\|_{L^{\infty}\left(\Omega_{1}\right)}+\left\|V_{x}\right\|\left\|3 u_{x x}-u_{y y}\right\|_{L^{\infty}\left(\Omega_{1}\right)}\right) \\
& +C\|V\|\left(h\|u\|_{H^{3}}+C h^{2}\|u\|_{H^{4}}\right) \\
\leq & C\left(h^{2-\sigma / 2}\left\|u_{x x}\right\|_{L^{\infty}\left(\Omega_{1}\right)}+h^{(5-\sigma-\alpha) / 2}\left\|3 u_{x x}-u_{y y}\right\|_{L^{\infty}\left(\Omega_{1}\right)}\right)\|V\| \\
& +C\left(h^{2}\|u\|_{H^{3}}+C h^{3}\|u\|_{H^{4}}\right)\|V\| .
\end{aligned}
$$

Together with the estimate (4.7), we have the following:

Theorem 2. For the model problem (4.14) with $u_{x y} \equiv 0$, the SDFEM on Peterson's meshes admits the following error estimate:

$$
\|u-U\| \leq C\left(h^{2}\|u\|_{H^{3}}+C h^{3}\|u\|_{H^{4}}\right)
$$

$+C \min \left\{\begin{array}{l}\left(h^{2-\sigma / 2}\left\|u_{x x}\right\|_{L^{\infty}\left(\Omega_{1}\right)}+h^{(5-\sigma-\alpha) / 2}\left\|3 u_{x x}-u_{y y}\right\|_{L^{\infty}\left(\Omega_{1}\right)}+h^{\alpha}\left\|u_{y y}\right\|\right) \\ h^{3 / 2}\left\|\nabla^{2} u\right\|\end{array}\right\}$.

Remark 4. Test computations show that the SDFEM for problem (4.14) has the superconvergence of order $O\left(h^{2}\right)$ if $3 u_{x x}-u_{y y} \equiv 0$. This means that the first term on the right-hand side of (4.15) should have a better convergence order. In view 
of the error behavior for the model problems, $V_{y}$ is continuous (which could not be proven). Then one can indeed use the trapezoidal rule to get an estimate like $O\left(h^{2}\|V\|\|u\|_{H^{4}}\right)$.

Remark 5. Based on the consideration of the above remark, we can optimize the choice of the artificial diffusion by taking $\alpha=(5-\sigma) / 3$. Then we can rewrite the convergence result as

$$
\|u-U\| \leq \begin{cases}C h^{(5-\sigma) / 3} & \text { for } 0 \leq \sigma \leq \frac{1}{2} \\ C h^{3 / 2} & \text { for } \frac{1}{2} \leq \sigma \leq 1\end{cases}
$$

Now, we consider a special mesh for $\sigma=1$ in which $m=h^{-1}$ exactly holds, i.e., we insert a vertical line at every nodal point on the $x$-axis in the original mesh. In this case, we rewrite the expansion formula (4.15) (assuming that $u_{y y} \equiv 0$ )

$$
\begin{aligned}
\left(\left(u-I_{h} u\right)_{y}, V_{y}\right)= & \frac{h^{2}}{8} \sum_{T}\left(\int_{\partial T_{12}} V_{x} u_{x x} d x-\int_{\partial T_{13}} V_{y} u_{x x} d y\right) \\
& +\frac{h^{2}}{8} \sum_{T}\left(\int_{\partial T_{24}} V_{y} u_{x x} d y-\int_{\partial T_{34}} V_{x} u_{x x} d x\right) \\
& +C\|V\|\left(h\|u\|_{H^{3}}+h^{2}\|u\|_{H^{4}}\right) .
\end{aligned}
$$

On integrating by parts in the $x$ - and $y$-direction, respectively, and on noting that $V u_{x x}$ is continuous at the nodal points, the nodal value $V\left(x_{m}, y_{n}\right) u_{x x}\left(x_{m}, y_{n}\right)$ arising from the integration disappears (see Figure 3 for notation). Thus, we obtain

$$
\begin{aligned}
\left(\left(u-I_{h} u\right)_{y}, V_{y}\right) & =\frac{h^{2}}{8} \sum_{T}\left(\int_{\partial T_{4}}\left(V u_{x x}\right)_{k} d s-\int_{\partial T_{1}}\left(V u_{x x}\right)_{k} d s\right) \\
& +\frac{h^{2}}{8} \sum_{T}\left(\int_{\partial T_{13}}-\int_{\partial T_{24}}\right) V u_{x x y} d y-\left(\int_{\partial T_{12}}-\int_{\partial T_{34}}\right) V u_{x x x} d x \\
& +C\|V\|\left(h\|u\|_{H^{3}}+h^{2}\|u\|_{H^{4}}\right) .
\end{aligned}
$$

From Figure 1 for $m=h^{-1}$ we see that all the sloping segments of type $\partial T_{1}$ and $\partial T_{4}$ make a line from boundary to boundary. Therefore, the first sum above disappears. The second sum has the same estimate as the third term. Combined with the estimate (4.7), this theoretically confirms the recovery of the superconvergence property of the SDFEM.

Figure 4 shows the dependence of the convergence order in the $L^{2}$-norm on the mesh parameter $\sigma$ for the numerical tests and for the theoretical analysis.

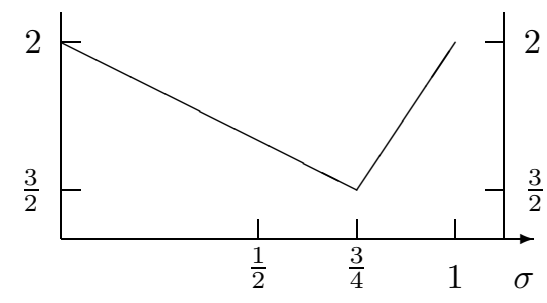

conv. order of tests

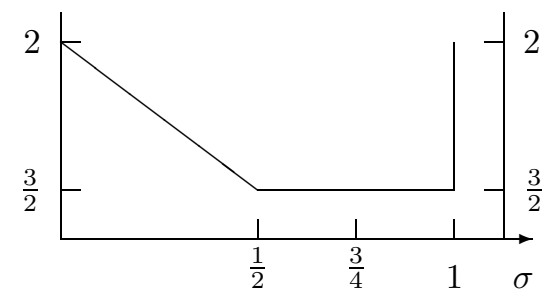

conv. order of analysis

Figure 4. Convergence order in the numerical tests and in the theoretical analysis 
Finally, let us consider the pointwise error estimates. For each point $\left(x_{0}, y_{0}\right) \in \Omega$, we define the discrete Green function $G \in \mathbf{V}_{h}^{0}$ by

$$
B(V, G)=V\left(x_{0}, y_{0}\right), \quad \forall V \in \mathbf{V}_{h}^{0} .
$$

Setting $V=I_{h} u-U$, we have

$$
V\left(x_{0}, y_{0}\right)=B(V, G)=B\left(I_{h} u-u, G\right)+\operatorname{Per}(u, G) .
$$

By virtue of a sharper estimate of the Green function $G$ given in [13] and the error expansions (4.8) and (4.11), we derive the following theorem.

Theorem 3. The SDFEM for problem (4.12) with $u_{x x} \equiv 0$ admits the error estimate

$$
\|u-U\|_{\infty} \leq C h^{2} .
$$

For problem (4.14), we define

$$
\Omega_{0}=\left\{(x, y): y-y_{0} \leq K h|\log h|,\left|x-x_{0}\right| \leq K h|\log h|\right\} .
$$

The following results can be obtained from the error expansions (4.7) and (4.15) by using the localizing technique in [13].

Theorem 4. For any $\nu \geq 2$, we can specify the constant $K=K(\nu)$ in (4.20) to fix $\Omega_{0}$. Suppose that the number of inserted vertical lines is $m \geq 1$ and that the exact solution of problem (4.14) satisfies

$$
u_{x y} \equiv 0, \quad u \in W^{4, \infty}\left(\Omega_{0}\right) .
$$

For $\left(x_{0}, y_{0}\right)$ away from any of the vertical lines, the SDFEM admits the error estimate

$$
\left|(u-U)\left(x_{0}, y_{0}\right)\right| \leq C h^{2}|\log h|\|u\|_{W^{4, \infty}\left(\Omega_{0}\right)}+C h^{\nu},
$$

and, for $\left(x_{0}, y_{0}\right)$ near the vertical lines,

$$
\begin{aligned}
\left|(u-U)\left(x_{0}, y_{0}\right)\right| \leq & C h^{3 / 2}|\log h|\left(\left\|u_{x x}\right\|_{L^{\infty}\left(\Omega_{0}\right)}+\left\|3 u_{x x}-u_{y y}\right\|_{L^{\infty}\left(\Omega_{0}\right)}\right) \\
& +C h^{2}|\log h|\|u\|_{W^{4, \infty}\left(\Omega_{0}\right)}+C h^{\nu} .
\end{aligned}
$$

\section{REFERENCES}

1. H. Blum, Qun Lin, R. Rannacher, Asymptotic error expansion and Richardson extrapolation for linear finite elements, Numer. Math. 49 (1986), 11-37. MR 87m:65172

2. T.J.R. Hughes, M. Mallet, A. Mizukami, A new finite element formulation for computational fluid dynamics: II. Beyond SUPG, Comput. Methods Appl. Mech. Engrg. 54 (1986), 341-355. MR 87f:76010b

3. C. Johnson, Numerical solution of partial differential equations by the finite element method, Cambridge University Press, Cambridge, 1987. MR 89b:65003a

4. C. Johnson, U. Nävert, An analysis of some finite element methods for advection-diffusion problems, in: Analytical and Numerical Approaches to Asymptotic Problems in Analysis (O. Axelsson et al., eds.), North-Holland, Amsterdam, 1981. MR 82e:65127

5. C. Johnson, A.H. Schatz, L.B. Wahlbin, Crosswind smear and pointwise errors in streamline diffusion finite element methods, Math. Comp. 49 (1987), 25-38. MR 88i:65130

6. Qun Lin, A rectangle test for finite element analysis, Proc. of Systems Science \& Systems Engineering, Great Wall (H.K.) Culture Publish Co., Hong Kong (1991), 213-216.

7. K. Niijima, Pointwise error estimates for a streamline diffusion finite element scheme, Numer. Math. 56 (1990), 707-719. MR 91h:65178

8. T.E. Peterson, A note on the convergence of the discontinuous Galerkin method for a scalar hyperbolic equation, SIAM J. Numer. Anal. 28 (1991), 133-140. MR 91m:65250 
9. G.R. Richter, An optimal-order error estimate for the discontinuous Galerkin method, Math. Comp. 50 (1988), 75-88. MR 88j:65197

10. B. Semper, Numerical crosswind smear in the streamline diffusion method, Comput. Methods Appl. Mech. Engrg. 113 (1994), 99-108. MR 94k:76060

11. Aihui Zhou, Qun Lin, Optimal and superconvergence estimates of the finite element method for a scalar hyperbolic equation, Acta Math. Sci.(Chinese) 14 (1994), 90-94. MR 95c:65161

12. Guohui Zhou, Local pointwise error estimates for the streamline diffusion method applied to nonstationary hyperbolic problems, East-West J. Numer. Math. 3 (1995), 217-235. MR 96g:65099

13. Guohui Zhou, R. Rannacher, Pointwise superconvergence of the streamline diffusion finite element method, Numerical Methods for Partial Differential Equations 12 (1996), 123-145. CMP 96:05

SFB 359 and Institut Für Angewandte Mathematik, Universität Heidelberg, Im Neuenheimer Feld 294, D-69120 Heidelberg, Germany

E-mail address: zhou@gaia.iwr.uni-heidelberg.de 